This is an electronic reprint of the original article. This reprint may differ from the original in pagination and typographic detail.

Author(s): Hu, Guanghui; Salo, Mikko; Vesalainen, Esa

Title: $\quad$ Shape identification in inverse medium scattering problems with a single far-field pattern

Year: $\quad 2016$

Version:

Please cite the original version:

Hu, G., Salo, M., \& Vesalainen, E. (2016). Shape identification in inverse medium scattering problems with a single far-field pattern. SIAM Journal on Mathematical Analysis, 48(1), 152-165. https://doi.org/10.1137/15M1032958

All material supplied via JYX is protected by copyright and other intellectual property rights, and duplication or sale of all or part of any of the repository collections is not permitted, except that material may be duplicated by you for your research use or educational purposes in electronic or print form. You must obtain permission for any other use. Electronic or print copies may not be offered, whether for sale or otherwise to anyone who is not an authorised user. 


\title{
Shape identification in inverse medium scattering problems with a single far-field pattern
}

\author{
Guanghui Hu*, Mikko Salo ${ }^{\dagger}$ Esa V. Vesalainen ${ }^{\ddagger}$
}

July 28,2015

\begin{abstract}
Consider time-harmonic acoustic scattering from a bounded penetrable obstacle $D \subset \mathbb{R}^{N}$ embedded in a homogeneous background medium. The index of refraction characterizing the material inside $D$ is supposed to be Hölder continuous near the corners. If $D \subset \mathbb{R}^{2}$ is a convex polygon, we prove that its shape and location can be uniquely determined by the far-field pattern incited by a single incident wave at a fixed frequency. In dimensions $N \geq 3$, the uniqueness applies to penetrable scatterers of rectangular type with additional assumptions on the smoothness of the contrast. Our arguments are motivated by recent studies on the absence of nonscattering wavenumbers in domains with corners. As a byproduct, we show that the smoothness conditions in previous corner scattering results are only required near the corners.
\end{abstract}

\section{Introduction and main results}

Assume a time-harmonic incident wave is incident onto a bounded penetrable obstacle $D \subset \mathbb{R}^{N}(N \geq 2)$ embedded in a homogeneous medium. The incident field $u^{\text {in }}$ may be any non-trivial solution in $L_{\text {loc }}^{2}\left(\mathbb{R}^{N}\right)$ of the Helmholtz equation

$$
\Delta u^{\text {in }}+k^{2} u^{\text {in }}=0 \quad \text { in } \quad \mathbb{R}^{N},
$$

where $k>0$ is the wavenumber. For instance, the incident wave is allowed to be a plane wave $\exp (i k x \cdot d)$ with incident direction $d \in \mathbb{S}^{N-1}:=\left\{x \in \mathbb{R}^{N}:|x|=1\right\}$, or a Herglotz wave of the form

$$
u^{\mathrm{in}}(x)=\int_{\mathbb{S}^{N-1}} \exp (i k x \cdot d) g(d) d s(d), \quad g \in L^{2}\left(\mathbb{S}^{N-1}\right) .
$$

\footnotetext{
${ }^{*}$ Weierstrass Institute, Mohrenstr. 39, 10117 Berlin, Germany. Email: hu@wias-berlin.de

${ }^{\dagger}$ University of Jyvaskyla, Department of Mathematics and Statistics, P.O. Box 35 (MaD), FI-40014 University of Jyvaskyla, Finland. Email: mikko.j.salo@jyu.fi

${ }^{\ddagger}$ University of Jyvaskyla, Department of Mathematics and Statistics, P.O. Box 35 (MaD), FI-40014 University of Jyvaskyla, Finland. Email: esavesalainen@gmail.com
} 
In this paper we suppose the scatterer $D$ to be a convex polygon in $\mathbb{R}^{2}$ or a convex polyhedron in $\mathbb{R}^{N}$. The physical properties of the inhomogeneous medium $D$ can be characterized by the refractive index function (or potential) $q(x)$. Without loss of generality we suppose $q(x)=1$ for $x \in D^{e}=\mathbb{R}^{N} \backslash \bar{D}$ due to the homogeneity of the background medium.

Denote by $u=u^{\text {in }}+u^{\text {sc }}$ the total field generated by $u^{\text {in }}$, where $u^{\text {sc }}$ is the outgoing scattered field which satisfies the Helmholtz equation $\left(\Delta+k^{2}\right) u^{\mathrm{sc}}=0$ in $D^{e}$ and the Sommerfeld radiation condition

$$
\lim _{|x| \rightarrow \infty}|x|^{\frac{N-1}{2}}\left\{\frac{\partial u^{\mathrm{sc}}}{\partial|x|}-i k u^{\mathrm{sc}}\right\}=0
$$

uniformly in all directions. The propagation of the total wave is governed by the Helmholtz equation

$$
\Delta u(x)+k^{2} q(x) u(x)=0 \quad \text { in } \quad \mathbb{R}^{N} .
$$

Across the interface $\partial D$, we assume the continuity of the total field and its normal derivative (already implicitly contained in the formulation (1.2)), i.e.,

$$
u^{+}=u^{-}, \quad \partial_{\nu} u^{+}=\partial_{\nu} u^{-} \quad \text { on } \partial D .
$$

Here the superscripts $(\cdot)^{ \pm}$stand for the limits taken from outside and inside, respectively, and $\nu \in \mathbb{S}^{N-1}$ is the unit normal on $\partial D$ pointing into $D^{e}$. The unique solvability of the scattering problem (1.1)-(1.3) in $H_{\text {loc }}^{2}\left(\mathbb{R}^{N}\right)$ is well-known if $q \in L^{\infty}\left(\mathbb{R}^{N}\right)$ (see e.g. [CK98, Chapter 8] or [Ki11, Chapter 6]). In particular, the Sommerfeld radiation condition (1.1) leads to the asymptotic expansion

$$
u^{\mathrm{sc}}(x)=\frac{e^{i k|x|}}{|x|^{(N-1) / 2}} u^{\infty}(\hat{x})+\mathcal{O}\left(\frac{1}{|x|^{N / 2}}\right), \quad|x| \rightarrow+\infty,
$$

uniformly in all directions $\hat{x}:=x /|x|, x \in \mathbb{R}^{N}$. The function $u^{\infty}(\hat{x})$ is a real-analytic function defined on $\mathbb{S}^{N-1}$ and is referred to as the far-field pattern or the scattering amplitude for $u^{\text {in }}$. The vector $\hat{x} \in \mathbb{S}^{N-1}$ is the observation direction of the far field.

This paper concerns the uniqueness in recovering the boundary $\partial D$ (or equivalently, the convex hull of the support of the contrast $q-1$ ) from the far-field pattern generated by one incident wave at a fixed frequency. The study on global uniqueness with a single incident plane or point source wave is usually difficult and challenging. For sound-soft or sound-hard obstacles, such uniqueness results have been obtained within the class of polyhedral or polygonal scatterers; see e.g., [AR05, CY03,EY06,EY08,HL14,LZ06]. The proofs rely heavily on the reflection principles for the Helmholtz equation with respect to a Dirichlet or Neumann hyperplane and on properties of the incident wave (for instance, plane waves do not decay at infinity and point source waves are singular). However, the approach of using reflection principles does not apply to penetrable scatterers due to the lack of "reflectible" (Dirichlet or Neumann) boundary conditions for the Helmholtz equation. To the best of our knowledge, uniqueness with one incident wave is still unknown within the class of non-convex polyhedral obstacles of impedance type. 
Earlier uniqueness results on shape identification in inverse medium scattering were derived by sending plane waves with infinitely many directions at a fixed frequency (see e.g., [EH11, Is08, Is90, KG08, Ki93]), which results in overdetermined inverse problems. Uniqueness with a single far-field pattern has been verified in two cases: $D$ is a ball (not necessarily centered at the origin) and $q \equiv q_{0} \neq 1$ is a constant in $D$ [HLL15], or $D$ is a convex polygon or polyhedron and $q$ is real-analytic on $\bar{D}$ satisfying $|q-1|>0$ on $\partial D$ [EH15]. The unique determination of a variable index of refraction $q$ in $\mathbb{R}^{N}$ from knowledge of the far-field patterns of all incident plane waves at fixed frequency, or by measuring the Dirichlet-to-Neumann map of the Helmholtz equation, has also been intensively studied. We refer to [SU87, HN87, Na88] and the survey [Uh14] for results for $N \geq 3$ and to recent results [Bu08, BIY15] for $N=2$.

The purpose of this article is to remove the real-analyticity assumption made in [EH15] on the refractive index. To do this, we employ a different method that is motivated by the recent studies [BPS14, PSV14] on the absence of non-scattering wavenumbers in corner domains. This method relies on the construction of suitable complex geometrical optics (CGO) solutions to the Helmholtz equation. Recall that $k$ is called a non-scattering wavenumber if there is a nontrivial incident wave whose far-field pattern vanishes identically. If $k$ is a non-scattering wavenumber, the functions $w=\left.u^{\text {in }}\right|_{D}$ and $\left.u\right|_{D}$ solve the interior transmission eigenvalue problem

$$
\left\{\begin{array}{lll}
\Delta w+k^{2} w=0, & \Delta u+k^{2} q u=0 & \text { in } \quad D \\
w=u, & \partial_{\nu} w=\partial_{\nu} u & \text { on } \partial D .
\end{array}\right.
$$

Thus each non-scattering wavenumber is an interior transmission eigenvalue (see the survey [CH13]). On the other hand, if $k^{2}$ is an interior transmission eigenvalue and if the non-trivial solution $w$ of (1.5) has a real-analytic extension from $D$ to $\mathbb{R}^{N}$, then $k^{2}$ is also a non-scattering wavenumber. This implies that, when $k^{2}$ is a non-scattering wavenumber, the Cauchy data of the total field $u$ on $\partial D$ coincide with the Cauchy data of a real-analytic function which satisfies the Helmholtz equation in a neighborhood of $D$. A similar phenomenon can be observed around a corner point, if two distinct convex polygons or polyhedra generate the same far-field pattern. Therefore, the argument for proving the absence of non-scattering wavenumber can be used for justifying uniqueness in determining the shape of a penetrable scatterer.

Let $D_{j}$ for $j=1,2$ be two penetrable scatterers with contrasts $q_{j}$. Denote by $u_{j}^{\infty}$ the far-field pattern of the scattered field caused by a fixed incoming wave $u^{\text {in }}$ incident onto $D_{j}$ with fixed wavenumber $k>0$. The first uniqueness result is in two dimensions, and applies to convex polygons.

Theorem 1.1. Let $D_{j} \subset \mathbb{R}^{2}$ for $j=1,2$ be bounded convex polygons. Assume that $q_{j} \in L^{\infty}\left(\mathbb{R}^{2}\right)$ are contrasts such that $q_{j} \equiv 1$ in $D_{j}^{e}$, and each vertex of $D_{j}$ has some neighborhood $U_{j}$ such that $\left.q_{j}\right|_{D_{j} \cap U_{j}}$ is $C^{\alpha}$ for some $\alpha>0$. Furthermore, assume that $q_{j}(O) \neq 1$ for each vertex $O$ of $D_{j}$. Then the relation $u_{1}^{\infty}=u_{2}^{\infty}$ on $\mathbb{S}^{1}$ implies that $D_{1}=D_{2}$.

The next result applies in dimensions $N \geq 3$ but requires that the scatterers are closed rectangular boxes, i.e. sets of the form $\left[0, a_{1}\right] \times \cdots \times\left[0, a_{N}\right]$ for some $a_{j}>0$ up 
to rotations and translations. We write $H^{s, p}$ for the fractional $L^{p}$ Sobolev space with smoothness index $s$.

Theorem 1.2. Let $D_{j} \subset \mathbb{R}^{N}$ for $j=1,2$ be two rectangular boxes. Assume that $q_{j} \in L^{\infty}\left(\mathbb{R}^{N}\right)$ are contrasts such that $q_{j} \equiv 1$ in $D_{j}^{e}$, and each corner of $D_{j}$ has some neighborhood $U_{j}$ such that $\left.q_{j}\right|_{\bar{D}_{j} \cap U_{j}}$ has regularity $X$ as specified below. Furthermore, assume that $q_{j}(O) \neq 1$ for each corner $O$ of $D_{j}$. Then the relation $u_{1}^{\infty}=u_{2}^{\infty}$ on $\mathbb{S}^{N-1}$ implies that $D_{1}=D_{2}$, provided that one of the following assumptions holds.

(a) $N=3$ and $X=C^{\alpha}$ for some $\alpha>1 / 4$.

(b) $N \geq 3$ and $X=H^{s, p}$ for some $s, p$ with $1<p \leq 2$ and $s>N / p$.

Theorems 1.1 and 1.2 are valid for Hölder or Sobolev potentials and avoid the realanalyticity assumption required in [EH15]. The results in dimensions $N \geq 3$ are confined to penetrable scatterers of rectangular type. It is still open how to prove Theorem 1.2 for general convex polyhedra with Hölder continuous contrasts. We remark that the above results remain valid for a large class of incident waves which do not vanish identically in a neighborhood of the scatterer. For instance, $u^{\text {in }}$ is also allowed to be a spherical point source emitted from some source position located in $D^{e}$.

Our technique improves the regularity conditions of the corner scattering results of [BPS14, PSV14]. Namely, regularity is only required in a small neighborhood of the corner point, and otherwise the contrasts are only required to be $L^{\infty}$. We state the results on the absence of non-scattering wavenumbers as follows. Throughout the paper we write $B_{r}:=\left\{x \in \mathbb{R}^{N}:|x|<r\right\}$ for $r>0$.

Theorem 1.3. Let $q \in L^{\infty}\left(\mathbb{R}^{2}\right)$, and let $W \subset \mathbb{R}^{2}$ be a closed sector with angle $<\pi$ and with vertex at $O$. Suppose that $q \equiv 1$ in $W^{e}$, that $q-1$ is compactly supported, and that $\left.q\right|_{W \cap B_{r}}$ is $C^{\alpha}$ for some $\alpha>0$ and $r>0$. Finally, assume that $q(O) \neq 1$. Then, with $q$ as the contrast, for any incoming wave $u^{\text {in }} \not \equiv 0$, the far-field pattern $u^{\infty}$ can not vanish identically.

Theorem 1.4. Let $q \in L^{\infty}\left(\mathbb{R}^{N}\right)$. Suppose that $q \equiv 1$ in $W^{e}$, that $q-1$ is compactly supported, and that $\left.q\right|_{W \cap B_{r}}$ has regularity $X$ for some $r>0$, where one of the following conditions holds:

(a) $N=3, W=\left[0, \infty\left[{ }^{3}\right.\right.$, and $X=C^{\alpha}$ for some $\alpha>1 / 4$.

(b) $N \geq 3, W=\left[0, \infty\left[{ }^{N}\right.\right.$, and $X=H^{s, p}$ for some $s, p$ with $1<p \leq 2$ and $s>N / p$.

Finally, assume that $q(O) \neq 1$. Then, with $q$ as the contrast, for any incoming wave $u^{\text {in }} \not \equiv 0$, the far-field pattern $u^{\infty}$ can not vanish identically.

The subsequent Section 2 is devoted to the proofs of the two-dimensional results, i.e., Theorems 1.1 and 1.3. The unique determination of a rectangular box in any dimension $N \geq 3$, Theorem 1.2, will be proved in Section 3. The result of Theorem 1.4 on nonscattering wavenumbers can be derived by using the same argument as in Theorem 1.3 and we omit its proof. 


\section{Proofs in two dimensions}

Denote by $(r, \varphi)$ the polar coordinates in $\mathbb{R}^{2}$, and by $B_{R}$ the disk centered at the origin $O$ with radius $R>0$. For $\varphi_{0} \in(0, \pi / 2)$, define $W \subset \mathbb{R}^{2}$ as the infinite sector between the half-lines $\Gamma^{ \pm}:=\left\{(r, \varphi): \varphi= \pm \varphi_{0}\right\}$. The closure of $W$ will be denoted by $\bar{W}$, which is a closed cone in $\mathbb{R}^{2}$. Set (see Figure 1 )

$$
S_{R}=W \cap B_{R}, \quad \Gamma_{R}^{ \pm}=\Gamma^{ \pm} \cap B_{R}, \quad \bar{S}_{R}=\bar{W} \cap B_{R}, \quad S_{R}^{e}=B_{R} \backslash \bar{S}_{R} .
$$

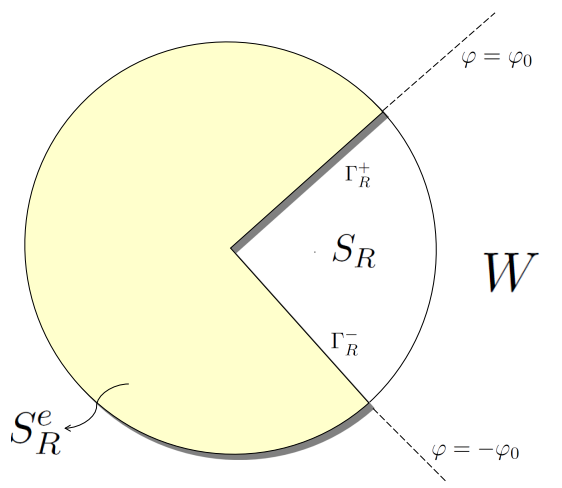

Figure 1: Geometrical settings.

The following two lemmas are the essential ingredients in the proofs. The first one concerns the construction of suitable Complex Geometrical Optics (CGO) solutions to the Schrödinger equation in $\mathbb{R}^{2}$. For convenience we employ the common notation $\langle x\rangle:=\left(1+|x|^{2}\right)^{1 / 2}$ throughout the paper.

Lemma 2.1. Let $\tilde{q} \in L^{\infty}\left(\mathbb{R}^{2}\right)$ satisfy $\tilde{q} \equiv 1$ in $\mathbb{R}^{2} \backslash \bar{W}$ and $\langle\cdot\rangle^{\beta}(\tilde{q}-1) \in C^{\alpha}(\bar{W})$ for some $\alpha>0$ and $\beta>5 / 3$. If $\rho \in \mathbb{C}^{2}$ satisfies $\rho \cdot \rho=-k^{2}$ and $|\operatorname{Im}(\rho)|$ is sufficiently large, then there exists a solution of the Helmholtz equation

$$
\Delta u(x)+k^{2} \tilde{q}(x) u(x)=0 \quad \text { in } \quad \mathbb{R}^{2}
$$

of the form

$$
u=e^{-\rho \cdot x}(1+\psi(x)),
$$

where $\psi$ satisfies

$$
\|\psi\|_{L^{6}\left(\mathbb{R}^{2}\right)}=\mathcal{O}\left(|\operatorname{Im}(\rho)|^{-1 / 3-\delta}\right) \quad \text { as } \quad|\rho| \rightarrow \infty,
$$

for some $\delta>0$.

Lemma 2.1 is the special case $N=2$ of Lemma 3.1 in [PSV14], the proof of which was based on the uniform Sobolev estimates of Kenig, Ruiz and Sogge [KRS87]. Relying on the construction of CGO solutions of the form (2.2), we next verify a result for the transmission problem between the Schrödinger equations with constant and piecewise Hölder continuous potentials in a finite polygonal cone. 
Lemma 2.2. Suppose $q \in L^{\infty}\left(B_{R}\right)$ satisfies $\left.q\right|_{\bar{S}_{R}} \in C^{\alpha}\left(\bar{S}_{R}\right)$ with some $\alpha>0$, and $q \equiv 1$ in $S_{R}^{e}$. Let $v_{1}, v_{2} \in H^{2}\left(B_{R}\right)$ be solutions to

$$
\Delta v_{1}(x)+k^{2} v_{1}(x)=0, \quad \Delta v_{2}(x)+k^{2} q(x) v_{2}(x)=0 \quad \text { in } \quad B_{R}
$$

subject to the transmission conditions

$$
v_{1}=v_{2}, \quad \partial_{\nu} v_{1}=\partial_{\nu} v_{2} \quad \text { on } \quad \Gamma_{R}^{ \pm} .
$$

Then we have $v_{1}=v_{2} \equiv 0$ in $B_{R}$ if $q(O) \neq 1$.

From Lemma 2.2 it follows that the Cauchy data of non-trivial solutions of the Schrödinger equations with constant and piecewise Hölder continuous potentials cannot coincide on two intersecting lines, if the potentials involved do not coincide on the intersection. The same result was verified in [EH15] but restricted to real-analytic potentials. Making use of classical corner regularity results for the Laplace equation in the plane (see e.g., [MNP00, Chapter 1.2], [Gr92, Chapter 2] or [Da88, Example 16.12]), the approach of Taylor expansion [EH15] can be generalized only to infinitely smooth potentials on $\bar{S}_{R}$. Hence, the above Lemma 2.2 has significantly relaxed the regularity assumption used in [EH15]. Below we carry out the proof of Lemma 2.2 which is valid only when the corner of $S_{R}$ is convex, i.e., $\varphi_{0}<\pi / 2$.

Proof of Lemma 2.2. We shall follow the approach from [PSV14, Section 4] but modified to be applicable to a polygonal convex cone with finite height. For clarity we divide the proof into three steps.

Step 1. Establish an orthogonality identity with an exponentially decaying remainder term. Set $w=v_{1}-v_{2}$. Then $w \in H^{2}\left(B_{R}\right)$, and we have

$$
\Delta w+k^{2} q w=k^{2}(q-1) v_{1} \quad \text { in } \quad B_{R}, \quad w=\partial_{\nu} w=0 \quad \text { on } \quad \Gamma_{R}^{ \pm} .
$$

Extending $q$ from $B_{R / 2}$ to $\mathbb{R}^{2}$ in a suitable way, we get a new potential $\tilde{q} \in L^{\infty}\left(\mathbb{R}^{2}\right)$ satisfying $\left.\tilde{q}\right|_{\bar{W}} \in C^{\alpha}(\bar{W})$ such that

$$
\tilde{q}=q \quad \text { in } \quad \bar{S}_{R / 2}, \quad \tilde{q} \equiv 1 \quad \text { in } \quad\left(\bar{W} \backslash \bar{S}_{R}\right) \cup\left(\mathbb{R}^{2} \backslash \bar{W}\right) .
$$

Clearly $\tilde{q}$ fulfills the assumptions in Lemma 2.1. Set $\beta:=\pi / 2-\varphi_{0}>0$. For any $\varphi$ with $\varphi \in]-\beta / 2, \beta / 2\left[\right.$, let $\omega=(\cos \varphi, \sin \varphi) \in \mathbb{S}^{1}$ and let $\omega_{ \pm}^{\perp}$ be the two vectors orthogonal to $\omega$, i.e., $\omega_{ \pm}^{\perp}= \pm(-\sin \varphi, \cos \varphi)$. For $\tau>0$, introduce the parameter-dependent vectors $\rho_{\tau, \varphi, \pm} \in \mathbb{C}^{2}$ as follows

$$
\rho_{\tau, \varphi, \pm}=\tau \omega+i\left(\tau^{2}+k^{2}\right)^{1 / 2} \omega_{ \pm}^{\perp}
$$

Obviously, $\rho_{\tau, \varphi, \pm} \cdot \rho_{\tau, \varphi, \pm}=-k^{2}$ and $\left|\rho_{\tau, \varphi, \pm}\right| \sim \sqrt{2} \tau$ as $\tau \rightarrow \infty$. By Lemma 2.1, we may construct solutions to the Schrödinger equation (2.1) of the form

$$
u(x)=u_{\tau, \varphi, \pm}(x)=\exp \left(-\rho_{\tau, \varphi, \pm} \cdot x\right)\left(1+\psi_{\tau, \varphi, \pm}(x)\right) \quad \text { in } \quad \mathbb{R}^{2}
$$


provided $\tau>0$ is sufficiently large. Applying Green's formula and using (2.5) yields

$$
\begin{aligned}
0 & =\int_{S_{R / 2}}\left(\Delta u+k^{2} \tilde{q} u\right) w d x \\
& =\int_{S_{R / 2}}\left(\Delta u+k^{2} q u\right) w d x \\
& =\int_{S_{R / 2}}\left(\Delta w+k^{2} q w\right) u d x+\int_{\partial\left(S_{R / 2}\right)}\left(\partial_{\nu} u w-\partial_{\nu} w u\right) d s \\
& =k^{2} \int_{S_{R / 2}}(q-1) v_{1} u d x+\int_{\Lambda_{R / 2}}\left(\partial_{\nu} u w-\partial_{\nu} w u\right) d s
\end{aligned}
$$

with $\Lambda_{R / 2}:=\{|x|=R / 2\} \cap W$. Since the constructed CGO solutions decay in $\bar{W} \backslash\{O\}$, we shall prove that the integral over $\Lambda_{R / 2}$ in (2.7) converges to zero exponentially fast as $\tau \rightarrow \infty$.

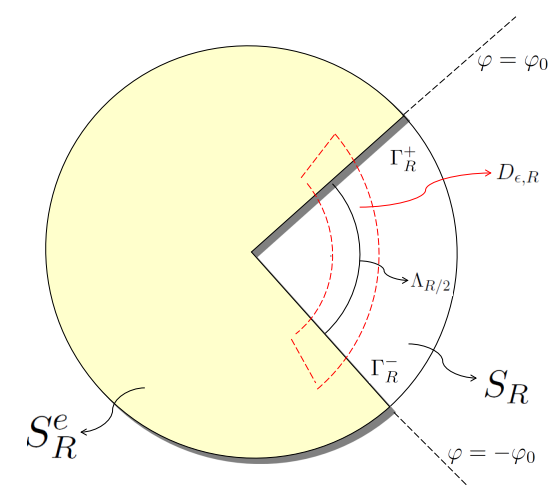

Figure 2: Configurations of $\Lambda_{R / 2}$ and $D_{\epsilon, R}$ in the proof of Lemma 2.2.

For $0<\epsilon<\min \{\beta / 2, R / 2\}$, define a neighborhood of $\Lambda_{R / 2}$ by (see Figure 2)

$$
D_{\epsilon, R}:=\left\{(r, \varphi): R / 2-\epsilon<r<R / 2+\epsilon,|\varphi|<\varphi_{0}+\epsilon\right\} .
$$

Then, there exists $\delta_{0}=\delta_{0}(\epsilon, R)>0$ such that

$$
\left.\operatorname{Re}\left(\rho_{\tau, \varphi, \pm} \cdot x\right)=\tau(\omega \cdot x) \geq \tau \delta_{0}>0 \quad \text { for all } \quad x \in D_{\epsilon, R}, \quad \varphi \in\right]-\beta / 2, \beta / 2[.
$$

This together with the estimates of $\left\|\psi_{\tau, \varphi, \pm}\right\|_{L^{6}\left(\mathbb{R}^{2}\right)}$ (see (2.3)) implies the exponential decay of the $L^{2}$-norm of $u_{\tau, \varphi, \pm}$ over $L^{2}\left(D_{\epsilon, R}\right)$, i.e.,

$$
\left\|u_{\tau, \varphi, \pm}\right\|_{L^{2}\left(D_{\epsilon, R}\right)}=\mathcal{O}\left(e^{-\tau \delta_{0}}\right) \quad \text { as } \quad \tau \rightarrow \infty .
$$

On the other hand, since $u_{\tau, \varphi, \pm}$ solves the Schrödinger equation in $\mathbb{R}^{2}$, the standard elliptic interior regularity estimate allows us to estimate for $\epsilon^{\prime}<\epsilon$ that

$$
\left\|u_{\tau, \varphi, \pm}\right\|_{H^{2}\left(D_{\epsilon^{\prime}, R}\right)} \leq C\left\|u_{\tau, \varphi, \pm}\right\|_{L^{2}\left(D_{\epsilon, R}\right)} \leq C e^{-\tau \delta_{0}} .
$$


Applying the Cauchy-Schwarz inequality and using the trace lemma, we may estimate the last term on the right-hand side of (2.7) as follows

$$
\begin{aligned}
\int_{\Lambda_{R / 2}}\left(\left(\partial_{\nu} u\right) w-\left(\partial_{\nu} w\right) u\right) d s & \leq C\|u\|_{H^{3 / 2}\left(\Lambda_{R / 2}\right)}\|w\|_{H^{3 / 2}\left(\Lambda_{R / 2}\right)} \\
& \leq C\|u\|_{H^{2}\left(D_{\epsilon^{\prime}, R}\right)}\left(\left\|v_{1}\right\|_{H^{2}\left(B_{R}\right)}+\left\|v_{2}\right\|_{H^{2}\left(B_{R}\right)}\right) .
\end{aligned}
$$

Combining (2.7) with the previous two inequalities, we get the following orthogonality identity over $S_{R / 2}$ with an exponentially decaying remainder term

$$
\left.\int_{S_{R / 2}}(q-1) v_{1} u_{\tau, \varphi, \pm} d x=\mathcal{O}\left(e^{-\tau \delta_{0}}\right) \quad \text { as } \quad \tau \rightarrow \infty, \quad \varphi \in\right]-\beta / 2, \beta / 2[.
$$

Step 2. Reduction to Laplace transforms. Assume that $v_{1} \not \equiv 0$. Since $v_{1}$ is a solution of the Helmholtz equation in $B_{R}$, the lowest order nontrivial homogeneous polynomial $H(x)$ in the Taylor expansion of $v_{1}$ around the origin is a harmonic function (see [BPS14, Lemma 2.4]). Without loss of generality, we assume $H$ is of order $n$ for some $n \geq 0$, i.e.,

$$
v_{1}(x)=H(x)+K(x), \quad K(x)=\mathcal{O}\left(|x|^{n+1}\right) \quad \text { as } \quad|x| \rightarrow 0 .
$$

Define $F$ to be the Laplace transform of $H$ in $W$,

$$
F(z):=\int_{W} \exp (-z \cdot x) H(x) d x
$$

for $z \in \mathbb{C}^{2}$ such that $\operatorname{Re}(z) \cdot(1,0)>\cos (\beta / 2)$. Taking $z=\rho=\rho_{\tau, \varphi, \pm}$ and splitting $F(\rho)$ into two terms, we see

$$
\begin{aligned}
F(\rho) & =\int_{S_{R / 2}} \exp (-\rho \cdot x) H(x) d x+\int_{W \backslash S_{R / 2}} \exp (-\rho \cdot x) H(x) d x \\
& =\int_{S_{R / 2}} \exp (-\rho \cdot x) H(x) d x+\mathcal{O}\left(e^{-\tau \delta_{1}}\right)
\end{aligned}
$$

as $\tau \rightarrow \infty$ for some $\delta_{1}=\delta_{1}\left(R, \varphi_{0}\right)>0$. By the assumption $q(O) \neq 1$, we may set $\eta:=q(O)-1 \neq 0$. Inserting (2.6) and (2.9) into (2.8) and then combining the resulting expression with (2.11) gives

$\eta F(\rho)=\int_{S_{R / 2}} \exp (-\rho \cdot x)(\eta H(x)-(q(x)-1)(H(x)+K(x))(1+\psi(x))) d x+\mathcal{O}\left(e^{-\tau \delta_{2}}\right)$ as $\tau \rightarrow \infty$, with $\delta_{2}:=\min \left\{\delta_{0}, \delta_{1}\right\}$. Making use of [BPS14, Lemma 3.6], we can estimate the integral on the right hand of the previous identity by (see e.g., [PSV14, Section 4])

$$
\begin{aligned}
& \int_{S_{R / 2}} \exp (-\rho \cdot x)(\eta H(x)-(q(x)-1)(H(x)+K(x))(1+\psi(x))) d x \\
= & \int_{S_{R / 2}} \exp (-\rho \cdot x)\{(q(O)-q(x)) H(x)-(q(x)-1)[K(x)+\psi(x)(H(x)+K(x))]\} d x \\
\leq & C \tau^{-n-2-\delta}
\end{aligned}
$$


for some $\delta>0$. Therefore, we arrive at

$$
\eta F(\rho) \leq C \tau^{-n-2-\delta}
$$

for all $\varphi \in]-\beta / 2, \beta / 2[$ and $\tau>0$ sufficiently large.

On the other hand, since the cone $W$ remains invariant under the transform $x \rightarrow|\rho| x$ and $H$ is a homogeneous polynomial, it is easy to check that

$$
F(\rho)=|\rho|^{-n-2} F(\rho /|\rho|) .
$$

Consequently, taking $\tau \rightarrow \infty$ in $(2.12)$ gives $F\left(\left(\omega+i \omega_{ \pm}^{\perp}\right) / \sqrt{2}\right)=0$. Moreover, the homogeneity of $F$ shown as in (2.13) yields

$$
\left.F\left(\tau\left(\omega+i \omega_{ \pm}^{\perp}\right)\right)=0 \quad \text { for all } \quad \tau>0, \quad \varphi \in\right]-\beta / 2, \beta / 2[.
$$

This implies the vanishing of the Laplace transform of $\chi_{W} H$ at $z=\tau\left(\omega+i \omega_{ \pm}^{\perp}\right)$ for all $\tau>0$ and $\varphi \in]-\beta / 2, \beta / 2[$.

Step 3. End of the proof. Repeating the arguments of [PSV14, Section 5], one can deduce from (2.14), taking both signs \pm , that $H \equiv 0$. This implies that $v_{1} \equiv 0$ in $B_{R}$. As a consequence, the Cauchy data of $v_{2}$ on $\Gamma_{R}^{ \pm}$vanish due to the transmission conditions (2.4). Finally, we get $v_{2} \equiv 0$ by the unique continuation of solutions to the Schrödinger equation. This finishes the proof of Lemma 2.2.

We are now ready to prove Theorems 1.1 and 1.3 for general incident waves, including point source waves.

Proof of Theorem 1.1. Since $u_{1}^{\infty}(\hat{x})=u_{2}^{\infty}(\hat{x})$ for all $\hat{x} \in \mathbb{S}^{1}$, applying Rellich's lemma we know that $u_{1}^{\mathrm{sc}}=u_{2}^{\mathrm{sc}}$ in $\mathbb{R}^{2} \backslash\left(\bar{D}_{1} \cup \bar{D}_{2}\right)$. Thus

$$
u_{1}(x)=u_{2}(x)
$$

for all $x \in \mathbb{R}^{2} \backslash\left(\bar{D}_{1} \cup \bar{D}_{2}\right)$.

If $\partial D_{1} \neq \partial D_{2}$, without loss of generality we may assume there exists a corner $O \in \mathbb{R}^{2}$ of $\partial D_{2}$ such that $O \notin \bar{D}_{1}$. We suppose further that this corner point coincides with the origin and we pick a fixed number $R>0$ such that $B_{R} \subset D_{1}^{e}$. Since $D_{2}$ is a convex polygon, rotating coordinate axes if necessary, we may assume that $D_{2} \cap B_{R}=\{(r, \varphi)$ : $\left.|\varphi|<\varphi_{0}\right\}$ for some $\varphi_{0} \in(0, \pi / 2)$; see Figure 3. From (2.15), it follows that

$$
u_{1}^{-}=u_{1}^{+}=u_{2}^{+}=u_{2}^{-}, \quad \partial_{\nu} u_{1}^{-}=\partial_{\nu} u_{1}^{+}=\partial_{\nu} u_{2}^{+}=\partial_{\nu} u_{2}^{-} \quad \text { on } \quad \partial D_{2} \cap B_{R},
$$

where the superscripts $(\cdot)^{-},(\cdot)^{+}$stand for the limits taken from $D_{2}$ and $D_{2}^{e}$, respectively. On the other hand, the function $u_{1}$ satisfies the Helmholtz equation with the wave number $k^{2}$ in $B_{R}$, while $u_{2}$ fulfills the Schrödinger equation

$$
\Delta u_{2}+k^{2} q_{2} u_{2}=0 \text { in } B_{R} .
$$

Since $q_{2}(O) \neq 1$, applying Lemma 2.2 leads to $u_{1}=u_{2} \equiv 0$ in $B_{R}$. Moreover, by unique continuation we obtain $u_{1}=u_{2} \equiv 0$ in $\mathbb{R}^{2}$. This implies that the scattered fields satisfy $u_{1}^{\mathrm{sc}}=u_{2}^{\mathrm{sc}}=-u^{\text {in }}$ in all of $\mathbb{R}^{2}$. Hence $u^{\text {in }} \equiv 0$ in $\mathbb{R}^{2} \backslash\left(\bar{D}_{1} \cup \bar{D}_{2}\right)$, but since $u^{\text {in }}$ solves the free Helmholtz equation, unique continuation implies $u^{\text {in }} \equiv 0$. This contradiction gives $D_{1}=D_{2}$. 


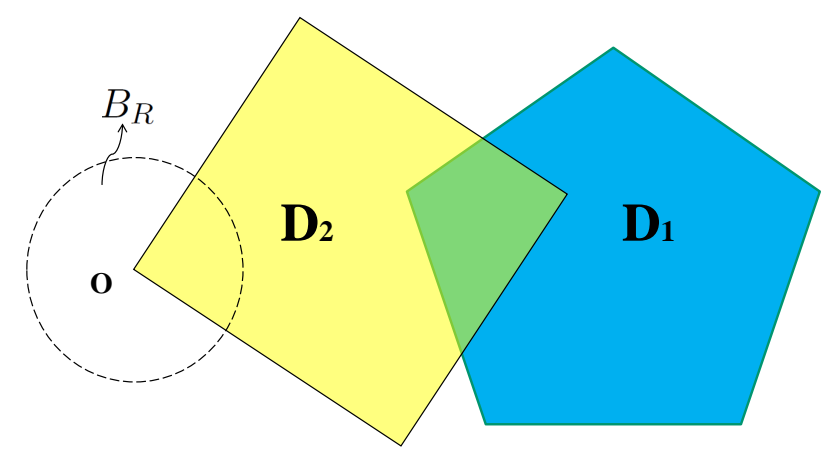

Figure 3: Two distinct convex penetrable scatterers $D_{1}$ and $D_{2}$ of polygonal-type.

Proof of Theorem 1.3. Let us consider the incident wave $u^{\text {in }} \not \equiv 0$ with the total wave $u$, so that we have

$$
\Delta u^{\text {in }}(x)+k^{2} u^{\text {in }}(x)=0 \quad \text { and } \quad \Delta u(x)+k^{2} q(x) u(x)=0 \quad \text { in } \mathbb{R}^{2} .
$$

If for this incident wave $u^{\infty} \equiv 0$, then Rellich's lemma tells us that $u \equiv u^{\text {in }}$ in $\mathbb{R}^{2} \backslash W$, and Lemma 2.2 applied with $v_{1}=u^{\text {in }}$ and $v_{2}=u$ gives $u^{\text {in }} \equiv 0$ in $B_{R}$. By unique continuation we get $u^{\text {in }} \equiv 0$, which is a contradiction.

\section{Proofs in higher dimensions}

We first present the proof under the assumption (a) of Theorem 1.2, that is, $D_{j} \subset \mathbb{R}^{3}$ is a rectangular box and the potential is $C^{\alpha}$ near the corners with $\alpha>1 / 4$. Let $W=\left[0, \infty\left[^{3}\right.\right.$. We will make use of the following result concerning complex geometrical optics solutions. Recall the notation $\langle x\rangle:=\left(1+|x|^{2}\right)^{1 / 2}$.

Lemma 3.1. Let $\widetilde{q} \equiv 1$ in $\mathbb{R}^{3} \backslash W$ and $\langle\cdot\rangle^{\beta}(\widetilde{q}-1) \in C^{\alpha}(W)$ for some $\alpha>1 / 4$ and $\beta>9 / 4$. If $\rho \in \mathbb{C}^{3}$ satisfies $\rho \cdot \rho=-k^{2}$ and $|\operatorname{Im}(\rho)|$ is sufficiently large, then there exists a solution of the Helmholtz equation

$$
\Delta u(x)+k^{2} \widetilde{q}(x) u(x)=0 \quad \text { in } \quad \mathbb{R}^{3}
$$

of the form

$$
u=e^{-\rho \cdot x}(1+\psi(x))
$$

where $\psi$ satisfies

$$
\|\psi\|_{L^{4}\left(\mathbb{R}^{3}\right)}=\mathcal{O}\left(|\operatorname{Im}(\rho)|^{-3 / 4-\delta}\right), \quad \text { as } \quad|\rho| \rightarrow \infty,
$$

for some $\delta>0$. 
The proof of the above lemma is also based on the uniform Sobolev estimates of Kenig, Ruiz and Sogge [KRS87]. In order to avoid repeating the arguments presented in [PSV14], we shall verify Lemma 3.1 by indicating the changes necessary to the proof of [PSV14, Theorem 3.1]. For this purpose we need to know into which Sobolev spaces the characteristic function of a cube belongs. Below we will write $\chi_{Q}$ for the characteristic function of a set $Q$, and denote by $C_{\mathrm{c}}^{\infty}\left(\mathbb{R}^{3}\right)$ the space of smooth functions with compact support.

Lemma 3.2. Let $Q \subset \mathbb{R}^{3}$ be a closed cube. Then

$$
\chi_{Q} \in H^{\tau, p}\left(\mathbb{R}^{3}\right)
$$

for $\tau \in[0,1 / 2[$ and $p \in] 1,2]$.

Proof. Without loss of generality, we consider $Q=[-1,1]^{3}$. Since $\chi_{Q} \in L^{1}\left(\mathbb{R}^{3}\right)$, the Fourier transform $\widehat{\chi Q}$ of $\chi_{Q}$ is continuous. For $\xi=\left(\xi_{1}, \xi_{2}, \xi_{3}\right) \in \mathbb{R}^{3}$ with $\xi_{1} \xi_{2} \xi_{3} \neq 0$, the transform $\widehat{\chi Q}$ takes the explicit form

$$
\widehat{\chi C}(\xi)=\frac{2^{3} \sin \xi_{1} \sin \xi_{2} \sin \xi_{3}}{\xi_{1} \xi_{2} \xi_{3}} .
$$

Thus, we may estimate

$$
\left\|\chi_{Q}\right\|_{H^{\tau, 2}\left(\mathbb{R}^{3}\right)}^{2}=\int_{\mathbb{R}^{3}}\langle\xi\rangle^{2 \tau}|\widehat{\chi C}(\xi)|^{2} \mathrm{~d} \xi \leq C \int_{\mathbb{R}^{3}}\langle\xi\rangle^{2 \tau}\left\langle\xi_{1}\right\rangle^{-2}\left\langle\xi_{2}\right\rangle^{-2}\left\langle\xi_{3}\right\rangle^{-2} \mathrm{~d} \xi .
$$

The last integral is finite when $\tau<1 / 2$. Thus, $\chi_{Q} \in H^{\tau, 2}\left(\mathbb{R}^{3}\right)$ for $\tau \in[0,1 / 2[$.

Next, let $\psi$ be a fixed function in $C_{\mathrm{c}}^{\infty}\left(\mathbb{R}^{3}\right)$ satisfying $\psi \chi_{Q}=\chi_{Q}$. By Hölder's inequality, for any $k \in\{0,1\}, f \in H^{k, 2}\left(\mathbb{R}^{3}\right)$ and fixed $\left.\left.p \in\right] 1,2\right]$,

$$
\|\psi f\|_{H^{k, p}\left(\mathbb{R}^{3}\right)} \leq C\|\psi f\|_{H^{k, 2}\left(\mathbb{R}^{3}\right)} \leq C\|f\|_{H^{k, 2}\left(\mathbb{R}^{3}\right)} .
$$

By interpolation, the mapping $f \mapsto \psi f$ maps $H^{s, 2}\left(\mathbb{R}^{3}\right)$ into $H^{s, p}\left(\mathbb{R}^{3}\right)$ for $\left.s \in\right] 0,1[$, and thus $\chi_{Q} \in H^{\tau, p}\left(\mathbb{R}^{3}\right)$ for all $\tau \in[0,1 / 2[$ and $p \in] 1,2]$.

The construction of CGO solutions for a cube is proved as follows.

Proof of Lemma 3.1. We may assume that $\alpha<1 / 2$. The proof of the complex geometric optics construction in [PSV14] is mostly independent of the shape of $W$. For $W=\left[0, \infty\left[^{3}\right.\right.$ we only need to check that $V:=\chi_{W}(1-\tilde{q})$ has the pointwise Sobolev multiplier property of Proposition 3.4 in [PSV14], i.e., we need to check that

$$
\|V f\|_{H^{\alpha-\varepsilon, 4 / 3}\left(\mathbb{R}^{3}\right)} \leq C\|f\|_{H^{\alpha-\varepsilon, 4}\left(\mathbb{R}^{3}\right)}
$$

for some constant $C>0$ for arbitrarily small fixed $\varepsilon>0$. The desired multiplier property in turn follows immediately, if we can show that (cf. [PSV14, Proposition 3.7])

$$
\langle\cdot\rangle^{-\gamma} \chi_{\left[0, \infty\left[{ }^{3}\right.\right.} \in H^{\tau, p}\left(\mathbb{R}^{3}\right)
$$


for $p \in] 1,2], \tau \in\left[0,1 / 2[\right.$ and $\gamma \in] 3 / p, \infty\left[\right.$. Given $\beta_{1}, \beta_{2} \in\left[0, \infty\left[\right.\right.$ with $\beta_{1}<\beta_{2}$, we set $\Lambda=\left[0, \beta_{2}\right]^{3} \backslash\left[0, \beta_{1}\left[^{3}\right.\right.$. Applying Lemma 3.2 we know that the function $\chi_{\Lambda}$ belongs to $H^{\tau, p}\left(\mathbb{R}^{3}\right)$. This leads to the relation (3.1) by changing variables and scaling the Sobolev norm; see the proof for Proposition 3.7 in [PSV14].

To continue the proof of Theorem 1.2 under the assumption (a), we again introduce some notation. Let $S_{R}=W \cap B_{R}, S_{R}^{e}=B_{R} \backslash S_{R}$, and $\Gamma_{R}=\partial W \cap B_{R}$. As in two dimensions, we will employ a result of the following type.

Lemma 3.3. Let $q \in L^{\infty}\left(B_{R}\right)$ satisfy $\left.q\right|_{\bar{S}_{R}} \in C^{\alpha}\left(\bar{S}_{R}\right)$, where $\alpha>1 / 4$, and $q \equiv 1$ in $S_{R}^{e}$. Let $v_{1}, v_{2} \in H^{2}\left(B_{R}\right)$ be solutions to

$$
\Delta v_{1}(x)+k^{2} v_{1}(x)=0 \quad \text { and } \quad \Delta v_{2}(x)+k^{2} q(x) v_{2}(x)=0 \quad \text { in } B_{R},
$$

subject to the transmission conditions

$$
v_{1}=v_{2}, \quad \partial_{\nu} v_{1}=\partial_{\nu} v_{2} \quad \text { on } \Gamma_{R}
$$

Then we have $v_{1}=v_{2} \equiv 0$ in $B_{R}$, if $q(O) \neq 1$.

Proof. We carry over the proof of Lemma 2.2 to three dimensions. Set $w=v_{1}-v_{2}$. Then we have $w=\partial_{\nu} w=0$ on $\Gamma_{R}$ and

$$
\Delta w(x)+k^{2} q(x) w(x)=k^{2}(q(x)-1) v_{1}(x) \quad \text { in } B_{R} .
$$

Extending $q$ from $B_{R / 2}$ to $\mathbb{R}^{3}$, we can obtain a new potential $\widetilde{q} \in C^{\alpha}(\bar{W})$ such that $\widetilde{q}=q$ in $\bar{S}_{R / 2}$ and that $\widetilde{q}-1$ satisfies the smoothness conditions required by Lemma 3.1. Next, write $\beta=\pi / 3$ and $a=\left(3^{-1 / 2}, 3^{-1 / 2}, 3^{-1 / 2}\right)$. Choose $\tau \in \mathbb{R}_{+}$and $\omega, \omega^{\perp} \in \mathbb{R}^{3}$ with $|\omega|=\left|\omega^{\perp}\right|=1, \omega \cdot \omega^{\perp}=0$ and $\omega \cdot a>\cos (\beta / 2)$. We parameterize the CGO solutions with the complex vector

$$
\rho=\rho_{\tau, \omega, \omega^{\perp}}=\tau \omega+i\left(\tau^{2}-k^{2}\right)^{1 / 2} \omega^{\perp} .
$$

Provided that $\tau$ is sufficiently large, Lemma 3.1 gives solutions

$$
u(x)=u_{\tau, \omega, \omega^{\perp}}(x)=e^{-\rho \cdot x}(1+\psi(x))
$$

to the Helmholtz equation $\Delta u(x)+k^{2} \widetilde{q}(x) u(x)=0$ in $\mathbb{R}^{3}$. Furthermore, the remainder $\psi$ has the $L^{4}$-estimate $\|\psi\|_{L^{4}\left(\mathbb{R}^{3}\right)}<C \tau^{-3 / 4-\delta}$ for some $\delta>0$. Arguing as before in the two-dimensional case, we get

$$
0=k^{2} \int_{S_{R / 2}}(q-1) v_{1} u d x+\int_{\Lambda_{R / 2}}\left(\left(\partial_{\nu} u\right) w-u \partial_{\nu} w\right) d s,
$$

where $\Lambda_{R / 2}=W \cap \partial B_{R / 2}$. The selection of the parameters $\beta, a$ and $\omega$ ensures the decay of the integral over $\Lambda_{R / 2}$,

$$
\int_{\Lambda_{R / 2}}\left(\left(\partial_{\nu} u\right) w-u \partial_{\nu} w\right) d s=O\left(e^{-\tau \delta_{0}}\right) \quad \text { as } \quad \tau \rightarrow \infty
$$


for some $\delta_{0}>0$. Thus, we again get the orthogonality relation

$$
\int_{S_{R / 2}}(q-1) v_{1} u d x=O\left(e^{-\tau \delta_{0}}\right) \quad \text { as } \quad \tau \rightarrow \infty
$$

for any given admissible $\omega$ and $\omega^{\perp}$.

Denote by $H$ the lowest degree nontrivial homogeneous polynomial in the Taylor expansion of $v_{1}$ around the origin, and consider the the Laplace transform $F(z)$ of $H$ (see $(2.10)$ ) for $z \in \mathbb{C}^{3}$ such that $\operatorname{Re}(z) \cdot a>\cos (\beta / 2)$. If $H$ has degree $n$, similarly as before we obtain $F(\rho)=O\left(\tau^{-n-3-\delta}\right)$ as $\tau \rightarrow \infty$, if $q(O) \neq 1$. This estimate involves using the Hölder inequality so that the $L^{4}$-norm of $\psi$ appears. In the other direction, by homogeneity, we have

$$
F(\rho)=|\rho|^{-n-3} F\left(\frac{\rho}{|\rho|}\right) .
$$

Taking $\tau \rightarrow \infty$ gives then $F\left(\tau \omega+i \tau \omega^{\perp}\right)=0$ for all $\tau \in \mathbb{R}_{+}$and all admissible $\omega$ and $\omega^{\perp}$. From [BPS14, Theorem 2.5] we obtain the conclusion $H(x) \equiv 0$, which implies $v_{1}=v_{2} \equiv 0$ in $B_{R}$.

Proof of Theorem 1.2 under assumption (a). The result follows by the same arguments as in the proof of Theorem 1.1, except that Lemma 3.3 is used instead of Lemma 2.2.

Next we indicate how to prove Theorem 1.2 under the assumption (b). Now we let $W=\left[0, \infty\left[{ }^{N}\right.\right.$ where $N \geq 3$. The required complex geometrical optics solutions were constructed in [BPS14] and they are given by the following result.

Lemma 3.4. Let $\widetilde{q} \equiv 1$ in $\mathbb{R}^{N} \backslash\left(W \cap B_{R}\right)$ for some $R>0$, and let $\left.\widetilde{q}\right|_{W \cap B_{R}}$ be in $H^{s, p}$ where $1<p \leq 2$ and $s>N / p$. Let also $D \subset \mathbb{R}^{N}$ be a bounded open set, and let $2 \leq r<\infty$. If $\rho \in \mathbb{C}^{N}$ satisfies $\rho \cdot \rho=0$ and $|\operatorname{Im}(\rho)|$ is sufficiently large, then there exists a solution of the Helmholtz equation

$$
\Delta u(x)+k^{2} \widetilde{q}(x) u(x)=0 \quad \text { in } \quad D
$$

of the form

$$
u=e^{-\rho \cdot x}(1+\psi(x))
$$

where $\psi$ satisfies

$$
\|\psi\|_{L^{r}(D)}=\mathcal{O}\left(|\operatorname{Im}(\rho)|^{-1}\right), \quad \text { as } \quad|\rho| \rightarrow \infty .
$$

Proof. We can write $\widetilde{q}=1-\chi_{K} \varphi$ for some cube $K=[0, a]^{N}$ and for some $\varphi \in H_{\mathrm{c}}^{s, p}\left(\mathbb{R}^{N}\right)$ by the conditions on $\widetilde{q}$ and the Sobolev extension theorem on Lipschitz domains. Writing $m=\chi_{K} \varphi$, the equation that we need to solve is

$$
\left(\Delta+k^{2}(1-m)\right) u=0 \quad \text { in } D .
$$

The result would then follow from [BPS14, Theorem 2.3], except that this theorem was proved under the condition $\varphi \in C^{\infty}$ instead of $\varphi \in H^{s, p}$. 
Inspecting the proof in [BPS14] we see that it is enough that the function

$$
Q=-k^{2}(1-m) \Phi_{D}
$$

where $\Phi_{D} \in C_{\mathrm{c}}^{\infty}\left(\mathbb{R}^{N}\right)$ satisfies $\Phi_{D}=1$ near $\bar{D}$, satisfies [BPS14, formula (34)], i.e. that one has

$$
Q \in \widehat{B_{r, 1}^{1}} \text { and }\|Q g\|_{\widehat{B_{r, 1}^{1}}} \leq C_{Q}\|g\|_{\widehat{B_{r, \infty}^{-1}}}
$$

where the spaces are as in [BPS14]. Now we can write $Q=Q_{1}+Q_{2}$ where $Q_{1}=$ $-k^{2} \Phi_{D} \in C_{\mathrm{c}}^{\infty}\left(\mathbb{R}^{N}\right)$ and $Q_{2}=f \chi_{K}$ where

$$
f=k^{2} \varphi \Phi_{D}
$$

so that $f \in H_{\mathrm{c}}^{s, p}\left(\mathbb{R}^{N}\right)$. We use [BPS14, Lemma 4.3 and preceding discussion] to conclude that when $\operatorname{supp}(q) \subset B_{R}$, one has

$$
\begin{aligned}
\|q\|_{\widehat{B_{r, 1}^{1}}} & \leq C R\|\hat{q}\|_{L^{r}}, \\
\|q g\|_{\widehat{B_{r, 1}^{1}}} & \leq 2 R^{2}\|\hat{q}\|_{L^{1}}\|g\|_{\widehat{B_{r, \infty}^{-1}}} \\
\left\|\chi_{K} g\right\|_{\widehat{B_{r, 1}^{1}}} & \leq C_{r}\|g\|_{\widehat{B_{r, 1}^{1}}}
\end{aligned}
$$

It follows that (3.2) will be satisfied if the function $f$ defined above satisfies $\hat{f} \in$ $L^{1} \cap L^{r}$. Since $f \in L_{\mathrm{c}}^{1}\left(\mathbb{R}^{N}\right)$, we have $\hat{f} \in L^{\infty}\left(\mathbb{R}^{N}\right)$ and it is enough to check that $\hat{f} \in L^{1}$. But if $\left(\psi_{j}(\xi)\right)_{j=0}^{\infty}$ is a Littlewood-Paley partition of unity and if $1 \leq p \leq 2$, we obtain by the Hölder and Hausdorff-Young inequalities that

$$
\begin{aligned}
\int_{\mathbb{R}^{N}}|\hat{f}(\xi)| d \xi & =\sum_{j=0}^{\infty} \int_{\mathbb{R}^{N}} \psi_{j}(\xi)|\hat{f}(\xi)| d \xi \leq C \sum_{j=0}^{\infty} 2^{j N / p}\left\|\psi_{j}(\xi) \hat{f}(\xi)\right\|_{L^{p^{\prime}}} \\
& \leq C \sum_{j=0}^{\infty} 2^{j N / p}\left\|\psi_{j}(D) f\right\|_{L^{p}} \leq C\|f\|_{B_{p, 1}^{N / p}}
\end{aligned}
$$

where $\psi_{j}(D)$ is the Fourier multiplier with symbol $\psi_{j}(\xi)$ and the last norm is a Besov norm. Since $H^{s, p} \subset B_{p, \infty}^{s} \subset B_{p, 1}^{N / p}$ for $s>N / p$, we get $\hat{f} \in L^{1}$ as required. This shows that (3.2) is satisfied, which concludes the proof.

Proof of Theorem 1.2 under assumption (b). It is enough to use Lemma 3.4 to prove an analogue of Lemma 3.3, and then argue as in the proof of Theorem 1.1.

\section{Concluding remarks}

In two dimensions, we have verified the uniqueness in identifying a convex penetrable scatterer of polygonal type with a single far-field pattern, provided the refractive index is discontinuous at the corner points but $C^{\alpha}$-Hölder continuous inside near the corners. 
In higher dimensions, the uniqueness applies to convex polyhedra with additional assumptions on the geometrical shape (i.e., boxes) and on the smoothness of the contrast. In this study, the smoothness assumption is required only near the corner points.

Our future efforts will be devoted to the uniqueness proof in 3D for convex polyhedra with general $C^{\alpha}$-Hölder $(\alpha>0)$ continuous potentials. Since the CGO solutions can be constructed with plenty of generality, the 3D proof essentially requires to evaluate the Laplace transform of a harmonic homogeneous polynomial over a general threedimensional corner domain and then to prove the vanishing of this polynomial through novel techniques. Another possible approach would be to analyze the corner and edge singularities of an elliptic equation with analytical Cauchy data in weighted Hölder spaces. Further results will be presented in a forthcoming publication.

\section{Acknowledgment}

The first author would like to acknowledge the support from the German Research Foundation (DFG) under Grant No. HU 2111/1-2. The second author was partly supported by an ERC Starting Grant (grant agreement no. 307023) and CNRS. The second and third authors were also supported by the Academy of Finland (Centre of Excellence in Inverse Problems Research), and they would like to thank the Institut Henri Poincaré Program on Inverse Problems in 2015 where part of this work was carried out.

\section{References}

[AR05] G. Alessandrini and L. Rondi, Determining a sound-soft polyhedral scatterer by a single far-field measurement, Proc. Amer. Math. Soc. , 133 (2005): 16851691 (Corrigendum: arXiv: math/0601406v1).

[BIY15] E. Blåsten, O. Yu. Imanuvilov, M. Yamamoto, Stability and uniqueness for a two-dimensional inverse boundary value problem for less regular potentials, Inverse Probl. Imaging (to appear).

[BPS14] E. Blåsten, L. Päivärinta and J. Sylvester, Corners always scatter, Comm. Math. Phys., 331 (2014): 725-753.

[Bu08] A. L. Bukhgeim, Recovering a potential from Cauchy data in the two dimensional case, J. Inverse Ill-Posed Probl., 16 (2008): 19-33.

[CH13] F. Cakoni and H. Haddar, Transmission eigenvalues in inverse scattering theory, in Inverse Problems and Applications: Inside Out II (edited by G. Uhlmann), MSRI Publications volume 60, Cambridge University Press, 2013.

[CY03] J. Cheng and M. Yamamoto, Uniqueness in an inverse scattering problem with non-trapping polygonal obstacles with at most two incoming waves, Inverse Problems, 19 (2003): 1361-1384 (Corrigendum: Inverse Problems 21 (2005): 1193). 
[CK98] D. Colton and R. Kress, Inverse Acoustic and Electromagnetic Scattering Theory, volume 93 of Applied Mathematical Sciences, Springer, New York, 1998.

[Da88] M. Dauge, Elliptic Boundary Value Problems in Corner Domains, Lecture Notes in Mathematics, Vol. 1341, Springer-Verlag, Berlin, 1988.

[EY06] J. Elschner and M. Yamamoto, Uniqueness in determining polygonal soundhard obstacles with a single incoming wave, Inverse Problems, 22 (2006): 355364 .

[EY08] J. Elschner and M. Yamamoto, Uniqueness in determining polyhedral soundhard obstacles with a single incoming wave, Inverse Problems, 24 (2008): 035004 .

[EH11] J. Elschner and G. Hu, Uniqueness in inverse transmission scattering problems for multilayered obstacles, Inverse Problems and Imaging, 5 (2011): 793-813.

[EH15] J. Elschner and G. Hu, Corners and edges always scatter, Inverse Problems, 31 (2015): 015003.

[Gr92] P. Grisvard, Singularities in Boundary Value Problems, Research Notes in Applied Mathematics, volume 22, Masson, Paris, 1992.

[HN87] G.M. Henkin, R.G. Novikov, The $\bar{\partial}$-equation in the multidimensional inverse scattering problem, Russian Math. Surveys, 42 (1987): 109-180.

[HLL15] G. Hu, J. Li and H. Liu, Uniqueness in determining refractive indices by formally determined far-field data, Appl. Anal., 94 (2015): 1259-1269.

[HL14] G. Hu and X. Liu, Unique determination of balls and polyhedral scatterers with a single point source wave, Inverse Problems, 30 (2014): 065010

[Is08] V. Isakov, On uniqueness of domains and general transmission conditions in inverse scattering, Comm. Math. Phys., 280 (2008): 843-858.

[Is90] V. Isakov, On uniqueness in the inverse transmission scattering problem, Comm. Partial Differential Equations, 15 (1990): 1565-1587.

[KRS87] C. E. Kenig, A. Ruiz, C. D. Sogge, Uniform Sobolev inequalities and unique continuation for second order constant coefficient differential operators, Duke Math. J., 55 (1987): 329-347.

[Ki11] A. Kirsch, An introduction to the mathematical theory of inverse problems, 2nd edition, volume 120 of Applied Mathematical Sciences, Springer, 2011.

[KG08] A. Kirsch and N. Grinberg, The Factorization Method for Inverse Problem$s$, volume 36 of Oxford Lecture Series in Mathematics and its Applications, Oxford University Press, Oxford, 2008.

[Ki93] A. Kirsch and R. Kress, Uniqueness in inverse obstacle scattering, Inverse Problems, 9 (1993): 285-299.

[LZ06] H. Liu and J. Zou, Uniqueness in an inverse obstacle scattering problem for both sound-hard and sound-soft polyhedral scatterers, Inverse Problems 22, (2006): 515-524. 
[MNP00] V. G. Maz'ya, S. A. Nazarov and B. A. Plamenevskii, Asymptotic Theory of Elliptic Boundary Value Problems in Singularly Perturbed Domains Volume I, Operator Theory: Advances and Applications Vol. 111, Birkhäuser-Verlag, Basel, 2000. Translated from the German by Georg Heinig and Christian Posthoff.

[Na88] A. Nachman, Reconstructions from boundary measurements, Ann. of Math., 128 (1988): 531-576.

[PSV14] L. Päivärinta, M. Salo and E. V. Vesalainen, Strictly convex corners scatter, arXiv:1404.2513.

[SU87] J. Sylvester and G. Uhlmann, A global uniqueness theorem for an inverse boundary value problem, Ann. of Math., 125 (1987): 153-169.

[Uh14] G. Uhlmann, Inverse problems: seeing the unseen, Bull. Math. Sci., 4 (2014): 209-279. 